\title{
Groove Extraction of Phonographic Records
}

\author{
Sylvain Stotzer ${ }^{1,2}$, Ottar Johnsen ${ }^{1}$, Frédéric Bapst ${ }^{1}$, and Rolf Ingold ${ }^{2}$ \\ ${ }^{1}$ University of Applied Sciences of Fribourg, CP 32 \\ 1705 Fribourg, Switzerland \\ \{sylvain.stotzer, ottar.johnsen, frederic.bapst\}@hefr.ch \\ www.eif.ch/visualaudio \\ ${ }^{2}$ DIVA Group, DIUF, University of Fribourg, Pérolles 2 - Bd de Pérolles 90, \\ 1700 Fribourg, Switzerland \\ rolf.ingold@unifr.ch
}

\begin{abstract}
Historical sound documents are of high importance for our cultural heritage. The sound of phonographic records is usually extracted by a stylus following the groove, but many old records are in such bad shape that no mechanical contact is possible. The only way to read them is by a contactless reading system. A phonographic document analysis system was developed using an optical technique to retrieve the sound from old records. The process is straightforward: we take a picture of each side of the disc using a dedicated analog camera, we store the film as our working copy, and when needed, we scan the film and process the image in order to extract the sound. In this paper, we analyze the imaging issues and present the algorithm for extracting the groove position and therefore the sound of the records.
\end{abstract}

\section{Introduction}

Cutting a disc was the only way to store sounds until the introduction of magnetic tape in the early 50's. Therefore there are huge collections of phonographic records, for example in radio stations and national sound archives. Such archives include pressed discs produced by record companies as well as direct cut discs obtained by the direct recording and available only as single copies with often a great cultural value. These discs are fragile and are deteriorating with time [1]. Worse, many records would be destroyed by the movement of the stylus from even the best turntables. Thus, we risk loosing an important cultural heritage.

On a turntable, a needle follows the groove's radial position. The radial velocity of the stylus is converted into an electrical signal corresponding to the sound. Therefore, the image of the record contains the sound information, as can be seen on Fig. 1. This observation has lead to the VisualAudio system:

1. An analog picture of a disc is taken, in order to preserve the sound information in case the original disc deteriorates.

2. When needed, the film is digitized using a specially designed rotating scanner.

3 . The sound is then extracted from the digital image by measuring the radial displacement of the groove. 


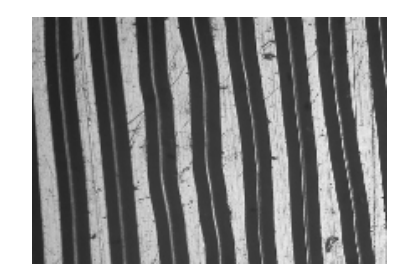

Fig. 1. Top view of a $78 \mathrm{rpm}$ record: the modulations of the groove are visible

Other optical techniques have been proposed to read phonographic records, but without the intermediate photographic step. Poliak et al presented an optical turntable using an optical fiber to follow the groove [2]. Such a technique is not suitable for severely damaged disc, where the groove has big discontinuities. Fadeyev et al. use methods derived from the particle physics instrumentation to recover audio data from musical cylinders and discs. They introduced a 2D imaging technique for $78 \mathrm{rpm}$ monaural discs, and a 3D surface measuring technique using confocal scanning microscopy [3].

The concept of VisualAudio has been introduced in [4], and we described the acquisition process and gave an estimation of the resolution and of the expected extracted signal quality [5], [6]. In this paper we analyze the image and processing issues for record pictures in VisualAudio. Section 2 outlines the image acquisition part of the system. Section 3 describes the groove and record images as well as the main alterations produced by the different steps of the acquisition process, leading to the edge model presented in section 4. Based on this model and on the knowledge of the phonographic record, we present the image processing algorithms used to extract the groove position in Sections 5, 6, 7 and 8. This paper focuses on monophonic 78 rpm (rotations per minute) records, as these are more fragile and more concerned by degradation and preservation issues. Differences between $78 \mathrm{rpm}$ and $331 / 3 \mathrm{rpm}$ record will be briefly exposed in Section 9. Finally Section 10 describes the results and system evaluation, followed by a brief conclusion in Section 11.

\section{The VisualAudio Image Acquisition}

The image acquisition of the VisualAudio system consists of two steps: the photography of the record, and scanning of the negative film. These stages are described in the next two subsections.

\subsection{Photography}

The groove position must be evaluated very accurately, requiring high image resolution. This can be done with microscope optics. Unfortunately the depth of the groove exceeds the microscope depth of field (DOF). Many discs also have a warping of up to $1 \mathrm{~mm}$. Using the intermediate photography step allows us to work with a larger DOF while imaging the disc, but ensures that the image to be digitized (the film) fits in the reduced DOF required by the microscope's optics. 
Most discs are black, and thus their surface has a very low reflectivity factor, as most of the light is absorbed. But since the discs are bright, their reflectivity is mainly specular, meaning that most of the reflected light has a reflective angle equal to the incidence angle. Thus we use a strong lighting that illuminates the disc uniformly from the lens point of view to ensure sufficient contrast between grooves and intergrooves.

Several kinds of cameras and films have been used to determine the equipment that best suits the specific needs of this project. A dedicated camera has been designed in order to satisfy high-resolution needs for record pictures [6].

\subsection{Scanning}

A dedicated rotating scanner has been built to digitize the record pictures [6]. The film lies on a glass turntable. The light source illuminates the film by transparency. A linear CCD camera is located above on the same axis as the light source and digitizes the film with 2048 sensors. On one rotation of the film, we scan a ring of the disc image (Fig. 2). By radial displacements of the camera, adjacent rings are scanned in order to digitize the whole record. The sampling rate of the camera combined with the turntable speed defines the image sampling frequency, ranging from 65 to $130 \mathrm{k}$ samples per ring. This frequency combined with the disc speed defines the sampling frequency of the output audio signal, ranging from 84 to $169 \mathrm{kHz}$ for $78 \mathrm{rpm}$ records.

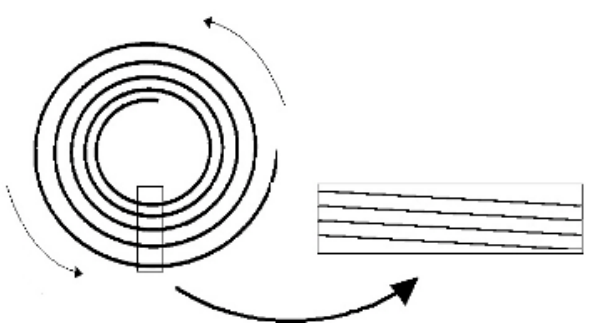

Fig. 2. The rotating scanner acquires one ring of the disc picture in a rectangular image file

As the camera is mounted on a 10x magnification optic and as the sensor size is $10 \mathrm{x} 10 \mu \mathrm{m}$, each captured pixel corresponds to a $1 \mathrm{x} 1 \mu \mathrm{m}$ area. As the glass tray rotates, each CCD-sensor integrates the light on a rotating length of the film, resulting in nonisometric pixels, which size varies from $1 \times 3.5 \mu \mathrm{m}$ up to $1 \times 15 \mu \mathrm{m}$ depending on the sampling rate and the radial position on the disc.

\subsection{Advantages of the VisualAudio Sound Extraction System}

The proposed sound extraction system will not replace standard turntables, but the photographic and image processing methods present numerous advantages over classical stylus playback:

- The optical method is contactless and can be applied on any record.

- Taking a picture of the discs is a quick way to store an analog copy of the sound content in its current state of conservation. 
- The document encoding is the same as on the original support: the groove displacement.

- The film is a stable medium, which can be preserved for more than hundred years.

- The ideal storage conditions for films and for discs are similar.

- The extraction process is independent of the recorded speed $\left(16,33^{1 / 3}, 78 \mathrm{rpm} ..\right)$ and of the material used to build the record.

- This extraction process is free of the transient noise produced by the mechanical resonance of the turntable arm on a damaged groove.

- The image of a groove shows several edges, which represent the same audio signal. This is very useful for advanced signal correction.

\section{Image Description}

\subsection{Groove Image}

As the light reflection on the disc is specular, the groove's shape has an important impact on the disc picture. During the photographic step, flat sections of the disc (intergroove) reflect the light perpendicularly to the camera objective and thus appear as black on the negative film. While the walls of the round monophonic grooves don't reflect light perpendicularly to the camera, the bottoms of the grooves do. The top edges between the intergrooves and the grooves are usually sharp and will result in a clear-cut luminance transition, but the bottom edges of the round shaped grooves will result in a blurred luminance transition, which is related to the groove curvature and the specular reflectance of the disc material [7]. Fig.3 displays an approximation of the 1-D intensity profile of a groove. A sample view of a negative picture of a record is shown on Fig 4.

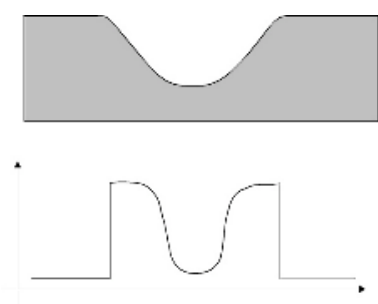

Fig. 3. $78 \mathrm{rpm}$ round shaped groove cut view and its approximate luminance model

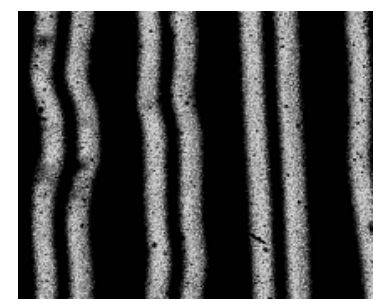

Fig. 4. $78 \mathrm{rpm}$ record photography on a negative film

\subsection{Image Degradations}

Several degradations may occur during the image acquisition process:

Needle damage: If the disc has been played on regular turntables, the groove's wall may have been damaged by the needle at some places. This produces flatter areas in the wall, leading in a darker trace on the photography of the groove (Fig. 5). 
Support degradations: Original support degradations (scratches, surface cracking...) or objects on the surface (dust, fungus...) may cause luminance change at the photographic step, causing trace cuts or spots on the acquired image.

Optics: The optical blur is produced by the out of focus blur and the Airy disk. Two separate optical systems are used in the VisualAudio process: the camera and scanner. Their resolutions have been described in details in [6]. The out of focus blur may vary on the whole record surface, due to the warping and the possible surface imperfections. Thus if the edge detection is not accurate enough, the optics blur may lead to a low frequency shift over the edge position. The out of focus blur may also be different at the top and the bottom of the groove.

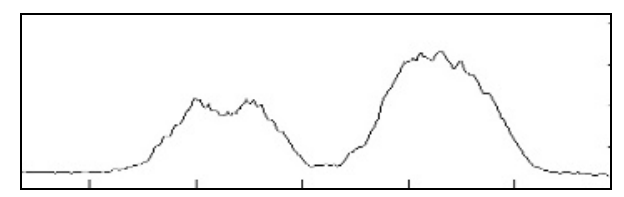

Fig. 5. Grey level intensity profile of a groove, as acquired by the VisualAudio scanner. The left wall has been damaged by the needle, which produced lower intensities in the middle of the white trace.

Motion blur: The motion blur is produced by the displacement of the groove relatively to the camera during the acquisition of a single sample. As the CCD camera is aligned with the center of the rotation glass tray, this motion blur appears only on the radial direction. The displacement due to the spiral and the off-axis are both smaller than $0.01 \mu \mathrm{m}$ and can be neglected [6]. Thus the motion blur is bounded by the maximum amplitude of the sound that modulates the groove:

$$
m_{\text {audio }}=\frac{V_{\max }}{n \cdot r p s}
$$

where $V_{\max }$ is the maximum recorded velocity, $n$ the number of samples per rotation and rps is the recorded speed in rotation per second. With a sampling frequency of $130 \mathrm{k}$-samples/rotation, and considering $\mathrm{V}_{\max }=32 \mathrm{~cm} / \mathrm{sec}$ for a $78 \mathrm{rpm}$ disc [8], then $m_{\text {audio }}=1.89 \mu \mathrm{m}$. As the motion blur is signal dependent, it is space-variant on the image. Thus if the edge detection is not precise, the motion blur may lead to a shift over the edge position that is proportional to the derivative of the signal.

\section{VisualAudio Groove Model}

As the sizes of the objects to detect (intergrooves, bottom and walls of the groove) are larger than the blur size, we assume that neighboring objects won't interfere in the edge detection process. Thus we may simplify the groove model and separate it in four distinct step edge models, having the same characteristics but not the same parameter values.

An 1-D edge model of an ideal object boundary could be represented by a step function $A u\left(x-x_{0}\right)+B$ where $x_{0}$ is the position of the edge, $A$ is the step amplitude, $B$ the base grey level and the unit step function $u(x)$ defined as [7]: 


$$
u(x)= \begin{cases}0 & \text { if } x \leq 0 \\ 1 & \text { otherwise }\end{cases}
$$

Blur on this edge is usually modeled by the Gaussian blurring kernel [9], [10]:

$$
g(x, \sigma)=\frac{1}{\sqrt{2 \pi} \sigma} e^{-x^{2} / 2 \sigma^{2}}
$$

where $\sigma$ is an unknown scale constant. Noise can be modeled as uncorrelated, zeromean, Gaussian distributed additive with a standard deviation $\gamma$ and could be integrated in the model as an additive parameter $n(x, \gamma)$.

We define $\sigma_{t}$ and $\sigma_{b}$ as the optical blur at the top respectively the bottom of the groove, and $\sigma_{m}$ the motion blur. Optical and motion blurs are space-dependent, but they may be considered as locally space-invariant. We assume that the groove bottom curvature is regular, defining $\sigma_{s}$ as the size of the blur due to the round shape of the groove bottom. The step amplitude $A$ and base level $B$ may vary between the two traces, taking values $A_{1}, A_{2}, B_{1}$ and $B_{2}$ (as seen on Fig. 5). These assumptions lead to the following 1-D edge models of a groove having its four edges $e_{1}, e_{2}, e_{3}$ and $e_{4}$ at positions $x_{1}, x_{2}, x_{3}$ and $x_{4}$ :

$$
\begin{aligned}
& e_{1}(x)=\left[A_{1} u\left(x-x_{1}\right)+B_{1}\right] * g\left(x, \sigma_{t}\right) * g\left(x, \sigma_{m}\right)+n(x, \gamma) \\
& e_{2}(x)=\left[A_{1}\left(1-u\left(x-x_{2}\right)\right)+B_{2}\right] * g\left(x, \sigma_{b}\right) * g\left(x, \sigma_{m}\right) * g\left(x, \sigma_{s}\right)+n(x, \gamma) \\
& e_{3}(x)=\left[A_{2} u\left(x-x_{3}\right)+B_{2}\right] * g\left(x, \sigma_{b}\right) * g\left(x, \sigma_{m}\right) * g\left(x, \sigma_{s}\right)+n(x, \gamma) \\
& e_{4}(x)=\left[A_{2}\left(1-u\left(x-x_{4}\right)\right)+B_{1}\right] * g\left(x, \sigma_{t}\right) * g\left(x, \sigma_{m}\right)+n(x, \gamma)
\end{aligned}
$$

If we rescale the step amplitudes to have unique values for $A$ and $B$, edges are symmetric two by two: the top $e_{1}$ and $e_{4}$, and the bottom of the groove $e_{2}$ and $e_{3}$.

\section{Image Analysis Algorithm}

The image analysis is performed on each dimension separately. Several reasons lead to a $1 \mathrm{D}$ edge detection process:

1. As pixels are non-isometric, we have much more accuracy for localization in the radial direction than on the tangential one.

2. The groove is perpendicular to the CCD camera during one sample acquisition. Small displacements of the groove are considered as motion blur (see Sect. 3.2)

3. Uses of the second dimension for the edge detection process would result in a low-pass filtering of the extracted sound signal.

Thus the edge detection process will be applied on each line of acquisition separately, and the process is time independent. The second direction information will be used later to detect and correct the signal in a post-processing step (see Sect. 8).

The image analysis algorithm is applied in three steps: first, the edge detection is performed on each line acquired by the camera. Then the groove reconstruction joins the extracted edges in the time dimension, and finally the detection and correction step is applied to cancel the damages and impulses on the extracted signal. 


\section{Edge Detection}

\subsection{VisualAudio Edge Detection Algorithm}

To retrieve the location of a single edge with high accuracy, we should detect the position of the middle of the transition between the luminance levels $A$ and $B$ in order to be independent from the space-varying blurs (i.e. optics blur and motion blur) over time. Unfortunately the step amplitude $A$ is difficult to retrieve due to the degradations explained in section 3. Therefore it will be almost impossible to locate the edge at the middle of the transition, thus producing a bias $b_{i}$ on the extracted signal that is signal dependent. But as the edges are symmetric two by two, the bias $b_{i}$ due to a space-variant degradation on an edge is compensated by the bias $-b_{i}$ on the symmetric edge. Thus the average of all edges of the trace produces negligible bias.

The edge detection is performed in two steps for each line captured by the camera: coarse and fine edge detection. These steps are detailed in the subsections 6.2 and 6.3.

\subsection{Coarse Edge Detection}

The purpose of the coarse edge detection is to detect the traces roughly on a smooth de-noised image. Thus we detect the presence of all the grooves and get an approximate location of the edges. Knowing these locations we can easily have a precise estimation of the local step amplitude $A$ and base value $B$, even in case of large luminance variation over the image. This is done by taking the maximal, respectively minimal, value between two neighboring detected traces, or a median estimation of these extremes. This coarse edge detection skips also part of the false detections that are induced by dust or damages, as it locates only objects having the desired size (the size of the traces). Coarse edge detection is implemented by a convolution with a double box filter $b(x)$ defined by a $\lambda x l$ kernel $\left[\begin{array}{lllllll}-1 & \ldots & -1 & 0 & 1 & \ldots & 1\end{array}\right]$. The result of this convolution is a smooth approximation of the derivative of the intensity profile (Fig. 6), which extremum locate the edges. As the $78 \mathrm{rpm}$ groove width and trace width $w$ are constant over the whole record, the scale of the double box filter is $\lambda=\alpha w$ with $\alpha$ being between 0.3 and 0.8 .

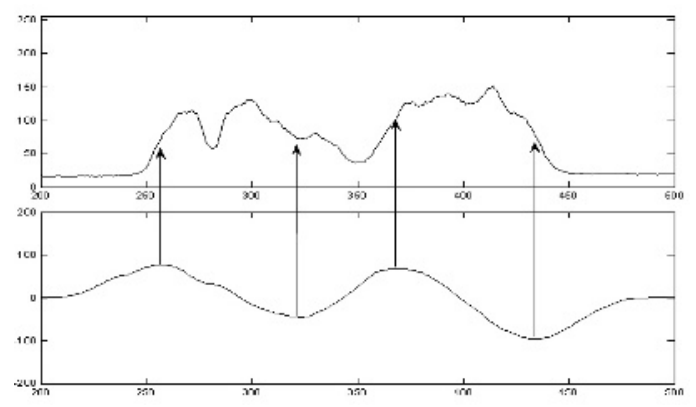

Fig. 6. Intensity profile of a groove (above) and the smooth derivative approximation obtained by convolution with the double box filter (below). Minima and maxima locate the edges roughly (arrows). 
Another way to detect approximately the traces over the image would be to initialize the process as described above at the beginning of the image processing, and then to propagate this information over time using the edges detected at the time $i$ to get the location of the edges at time $i+1$. However, due to scratches or other physical damage of the record, trace cuts may occur all over on the image (even during the initialization step) and lead to the loss of some grooves. Such time propagated information has also the disadvantage to propagate errors. Therefore choice has been made to have this coarse scale edge detection at each sampling time.

\subsection{Fine Edge Detection}

The purpose of the fine edge detection is to locate the edge accurately. One of the constraints given by the model is to detect the position relatively to the local step amplitude $A$ and base value $B$ to keep the edges symmetric two by two. The simplest method to satisfy this constraint is to use an adaptive threshold $t$ defined as follows:

$$
t=B+\beta A, \quad \beta \in[0,1]
$$

In practice $\beta$ should be restricted to the $[0.2,0.7]$ in order to be noise robust. As the white areas (high amplitude on the intensity profile of Fig. 6) are noisier, a low $\beta$ value produces results that are more robust to noise and degradations. Subpixel accuracy is then achieved by linear interpolation between the two consecutives pixels $p(x)$ and $p(x+1)$ which satisfy $p(x) \geq t$ and $p(x+1) \leq t$ (or the opposite).

The white traces are noisier than the intergroove and may contain some darker areas that could mislead the edge detection. To avoid such false detection, the thresholding process is applied starting from the intergroove or from the bottom of the groove. Edges are then located at the first major discontinuities higher than $t$ (Fig. 7).

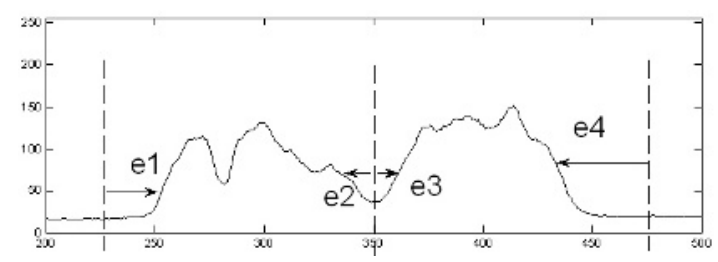

Fig. 7. Edges are found by thresholding. To avoid false detection, the fine edge detection is processed starting from the intergroove and bottom of groove locations (dashed lines).

More sophisticated thresholding methods using linear approximation or parametric fitting of the luminance transition have been tested. The results were similar to this method. This can be explained by the strong blur that smoothes the image, producing the same positioning accuracy with this simple method.

\section{Groove Reconstruction}

Once the 1-D edge detection is performed in the radial direction, detected edge positions must be put in a row to rebuild the recorded signal in the time, 
corresponding to the $2^{\text {nd }}$ dimension of the image. These vectors will be defined as the extracted sound signals $s_{1}, s_{2}, s_{3}$ and $s_{4}$ retrieved from the four edges of the groove.

As we know the position $s_{i}(j)$ of an edge $i$ at time $j$ and as we know the maximal displacement of the groove (equation (1)), we may define a margin for the edge at time $j+1$. This margin is defined for each edge at each sampling time and does not overlap with the neighboring traces margins at time $i$. The edge position $s_{i}(j+1)$ is then chosen among all the candidates inside the margin to minimize either :

$$
\left|s_{i}(j)-s_{i}(j+1)\right| \quad \text { or } \quad\left|2 s_{i}(j)-s_{i}(j-1)-s_{i}(j+1)\right|
$$

Thus the use of these margins is a first rough denoising process. Most of the time, candidates are unique inside a margin, but these margins are a helpful tool to follow the trace in case of a cut or damaged trace. The margin displacement depends on the signal and the sampling frequency. In case of trace cuts, the margin displacement is driven either by the displacement of:

a) the other edge of the same trace (if existing).

b) the edges of the other trace of the same groove.

c) all the other traces in the image.

The average of all the other traces displacement will result in an approximation of the low frequencies component of the signal, i.e. the spiral of the record and the off-axis due to the bad centering of the film on the scanner.

Finally the relative position of each groove is quantified by the sum its four edges.

\section{Edge Correction}

Some samples of the extracted signals $s_{i}$ cannot be defined by the edge detection and groove construction steps. Moreover some samples are corrupted and must be corrected according to the neighboring samples. To solve these issues we use a corrupted samples detection/correction scheme.

In order to be signal independent, the corrupted samples detection is applied on the second derivative $\partial^{2} s_{i}$ of the extracted signal $s_{i}$. The detection is performed using a double-threshold method [11]. The high threshold $\tau_{\mathrm{h}}$ is used to detect the impulses and the lower threshold $\tau_{1}$ to estimate the impulsion duration and to determine the samples to correct. Both $\tau_{h}$ and $\tau_{1}$ are defined using the standard deviation $\sigma$ of $\partial^{2} s_{i}$ defined on a local window, typically over 1000 samples:

$$
\tau_{h}=a \sigma \text { and } \tau_{l}=b \sigma \quad \text { with } a>b>1
$$

The factors values are usually about $a=4$ and $b=2$. The corrupted samples detection process is then applied in two steps:

1. Detect the samples $j$ with $\partial^{2} s_{i}(j)>\tau_{h}$

2. Starting from $j$, all the contiguous samples with $\partial^{2} s_{i}(k)>\tau_{l}$ are marked as corrupted.

The corrupted samples correction is performed either by interpolation or by using the other edges information. As the signal is oversampled and the displacement of the groove over consecutive time samples has relatively small amplitude, short 
corrections up to 5 samples (which is much smaller than the wavelength of the highest recorded frequency) are performed by interpolation using first or second order polynomials.

In case of physical damages on the record, the sound signal can be extracted using only the information from the undamaged edges and traces. Most of the scratches are not perpendicular to the groove, meaning that the extracted signal from the different edges will be damaged at different sampling time. Thus correct value of the corrupted samples can be estimated by using the other edges. To avoid the effect of the edge detection bias on the signal, we must cancel this bias by using the following combination of $s_{2}, s_{3}$ and $s_{4}$ to replace a damaged area on edge $s_{1}$ :

$$
s_{1}=s_{2}+s_{3}-s_{4}
$$

\section{Groove Extraction Comparison Between $78 \mathrm{rpm}$ and 331/3 rpm}

The technology improvement of the $33 \frac{1}{3} \mathrm{rpm}$ microgroove over the $78 \mathrm{rpm}$ leads to several differences in the image of a record. Those affect the signal to noise ratio of the extracted sound from $33 \frac{1}{3} \mathrm{rpm}\left(\mathrm{SNR}_{33}\right)$ in comparison with the $\mathrm{SNR}_{78}$ as follows:

- $33 \frac{1}{3} \mathrm{rpm}$ grooves have a triangular shape. The consequence is that the bottom of the groove is not visible on the acquired image, and that we get only one trace to represent each groove. This limits the quality of the extraction and correction steps. As the extracted signal is averaged using two edges instead of four, the $\mathrm{SNR}_{33}$ is then $3 \mathrm{~dB}$ lower.

- The $33 \frac{1}{3} \mathrm{rpm}$ microgrooves have less deviation, meaning that we get less dynamic for the displacement measurement. As the $78 \mathrm{rpm}$ groove deviation is three times bigger than on $331 / 3 \mathrm{rpm}$ records, $\mathrm{SNR}_{33}$ is $20 \log _{10}(3)=9.5 \mathrm{~dB}$ lower.

- With the $33 \frac{1}{3} \mathrm{rpm}$ records, the in-band noise is enhance by a factor $78 / 33$ compared to the $78 \mathrm{rpm}$, as the speed increase puts more noise out of band. Thus the $\mathrm{SNR}_{33}$ is then $3.7 \mathrm{~dB}$ lower.

Therefore the sound extraction for $33 \frac{1}{3} \mathrm{rpm}$ results in lower quality than for $78 \mathrm{rpm}$ :

$$
S N R_{33}=S N R_{78}-16.2 \mathrm{~dB}
$$

This is not an issue as $33 \frac{1}{3} \mathrm{rpm}$ are less concerned by preservation. However the reading of stereo microgroove $33^{1 / 3} \mathrm{rpm}$ is also possible with the VisualAudio system in order to have a complete system and to be able to read as many records as possible. For example test records are a very useful tool to measure the quality of a sound extraction system, but they are almost only available on $331 \frac{1}{3} \mathrm{rpm}$.

\section{Evaluation and Results}

\subsection{Edge Detection Quality}

The precision of the edge detection scheme can be estimated by measuring the residual noise on the signal extracted from an unmodulated groove. The advantage of 
this kind of test is that it can be performed on any record as such unmodulated grooves can be found at the beginning and at the end of each track.

To evaluate the accuracy of the edge detection process, differences were measured between consecutive detected edges positions on unmodulated grooves. The standard deviation of these measurements reaches 0.5 pixels $(=0.5 \mu \mathrm{m})$ for $33 \mathrm{rpm}$ records and 0.3 pixels for $78 \mathrm{rpm}$ records. This demonstrates that the groove extraction reaches subpixel accuracy and that the resolution of this system is sufficient to extract good quality sound from the image of phonographic records.

\subsection{Quality of the Extracted Signal}

$33 \frac{1}{3} \mathrm{rpm}$ test records contain sinewave tracks used to evaluate the tracking ability. These tracks can be used in our system to measure a signal to noise ratio (SNR), as well as to detect the presence of harmonics and intermodulations.

Current SNR measurements reach $36 \mathrm{~dB}$ for the extraction of a $300 \mathrm{~Hz}$ track on a test record. As this track is amplified by $15 \mathrm{~dB}$, the $S N R_{33}$ for a non-amplified record would be $21 \mathrm{~dB}$. Therefore we can estimate the $S N R_{78}$ using equation (9):

$$
S N R_{78}=S N R_{33}+16.2 d B=37.2 d B
$$

This estimation of $S N R_{78}$ is comparable to the recorded quality of the $78 \mathrm{rpm}$.

Several harmonics may also be present in the sound extraction of a sinewave. The amplitude of the second harmonic gives information about the edge detection bias. The extraction results presented on Table 1 have been performed on a $300 \mathrm{~Hz}$ frequency extracted from a $33 \frac{1}{3} \mathrm{rpm}$ test record. The output level is given in $\mathrm{dB}$ for each extracted edge, as well as for the sum of both edges. The sum enhances the signal by $6 \mathrm{~dB}$ over each edge, which corresponds to the expected gain for amplification by a factor 2 . On the other hand, the sum of both edges reduces the $2^{\text {nd }}$ harmonic by $16 \mathrm{~dB}$. This means that part of the $2^{\text {nd }}$ harmonic is due to a symmetric phenomenon that appears on both edges and which is canceled by summing both signals. Different values for the threshold $\beta$ lead to similar results and observations.

Table 1. Output level for a $300 \mathrm{~Hz}$ track extraction using an adaptive threshold with $\beta=0.2$

\begin{tabular}{|l|l|l|l|}
\hline & Edge 1 & Edge 2 & Edge1+Edge2 \\
\hline Signal $(300 \mathrm{~Hz})$ & $-63 \mathrm{~dB}$ & $-64 \mathrm{~dB}$ & $-57 \mathrm{~dB}$ \\
\hline $2^{\text {nd }}$ harmonic $(600 \mathrm{~Hz})$ & $-93 \mathrm{~dB}$ & $-92 \mathrm{~dB}$ & $-109 \mathrm{~dB}$ \\
\hline
\end{tabular}

\section{Conclusion}

Based on the described process, a photography camera, dedicated scanner prototype and extraction software have been developed. This system is fully functional and has demonstrated that we can take photography of a record and extract the sound out of the picture by image processing techniques. The extracted sound quality for $78 \mathrm{rpm}$ records is comparable to the sound of a turntable. It is also possible to extract sound from records damaged by scratches or affected by fungus. The reading of more severely damaged records is still under development, as for example broken records or direct cut disc with shrinkage of the recording layer. 
Collaborations with sound archives are currently starting, and the VisualAudio system should begin shortly to preserve the sound from large collections of old phonographic records.

Several recovered sound samples can be found at www.eif.ch/visualaudio.

\section{Acknowledgements}

The authors thank C. Milan and T. Fumey for their help in this project.

This project is funded by the Gebert Rüf Foundation (project P024/03) and the Swiss National Science Foundation (project 21-64984.01).

\section{References}

1. Bradley, K. et al: Guidelines on the Production and Preservation of Digital Audio Objects. IASA - TC04 (2004) ISBN 87-990309-1-8

2. Poliak, J., Robert, P., Goy, J.: Optical Fibre Turntable for Archive Records, Proceedings of the $92^{\text {nd }}$ Convention AES, Vienna, Austria (1992)

3. Fadeyev, V. , Haber, C.: Reconstruction of Mechanically Recorded Sound by Image Processing, J. Audio Eng. Soc. (2003) 51(12): 1172-1185

4. Cavaglieri, S., Johnsen, O., Bapst, F.: Optical Retrieval and Storage of Analog Sound Recordings. AES $20^{\text {th }}$ International Conference, Budapest (2001)

5. Stotzer, S., Johnsen, O., Bapst, F., Sudan, C., Ingold, R.: Phonographic Sound Extraction Using Image and Signal Processing, IEEE Proceedings 2004 ICASSP (2004) 4:289-292.

6. Stotzer, S., Johnsen, O., Bapst, F., Milan, C., Ingold, R.: Phonographic Sound Extraction using Photography and Signal Processing, Digital Signal Processing, Elsevier, to be published

7. Elder, J.: The Visual Computation of Bounding Contours. PhD Thesis, Mc Gill University, Dept. of Electrical Engineering (1995)

8. Temmer, S.F. (ed.): Disk Recording Volume 1 (Anthology), Audio Engineering Society, New York (1980)

9. Canny, J.: A computational approach to edge detection. IEEE Trans. on Pattern Analysis and Machine Intelligence (1986) 8(6):679-698

10. Pentland, A.P.: A new sense for depth of field. IEEE Tran. PAMI (1987) 9(4):523-531

11. Esquef, P., Biscainho, L.W.P., Diniz, P.S.R., Freeland, F. P.: A double threshold-based approach to impulsive noise detection in audio signal. Proc. X European Signal Processing Conf. (EUSIPCO 2000), Tampere, Finland (2000) 2041-2044 